\title{
Effect of wearing masks on postoperative radiotherapy in cervical cancer patients during the COVID-19 pandemic: a retrospective study on acute toxicity
}

\section{Guangyu Zhang}

Qilu Hospital of Shandong University

\section{Cong Wang}

Shandong Cancer Hospital and Institute, Shandong First Medical University, Shandong Academy of Medical Sciences

\section{Changdong $\mathrm{Ma}$}

Qilu Hospital of Shandong University

\section{Li Miao}

Qilu Hospital of Shandong University

\section{Youzhong Zhang}

QiLu Hospital of Shandong University

Chunli Fu ( $\square$ ffcl2001@outlook.com )

Qilu Hospital of Shandong University

\section{Research Article}

Keywords: COVID-19, cervical cancer, mask, concurrent chemoradiotherapy, toxicity

Posted Date: February 8th, 2022

DOI: https://doi.org/10.21203/rs.3.rs-1330420/v1

License: (c) (i) This work is licensed under a Creative Commons Attribution 4.0 International License. Read Full License 


\section{Abstract}

Background: The COVID-19 pandemic has created great challenges and requirements for radiotherapy. Mask wearing is an effective physical intervention against disease transmission and has been part of daily prevention strategies. The aim of the study was to evaluate the effect of mask wearing on the toxicity of postoperative radiotherapy in patients with cervical cancer.

Methods: Between December 2018 and June 2021, a retrospective review was conducted among patients with FIGO stage IB-IIA cervical cancer who were treated with postoperative concurrent chemoradiotherapy. Acute toxicities were graded according to the National Cancer Institute's Common Terminology Criteria for Adverse Events, version 3.0.

Results: A total of 85 patients were eligible for the study; 33 patients (38.9\%) wore masks, whereas another 52 patients $(61.1 \%)$ did not wear masks. There was a statistically significant difference in the incidence of grade $1-2$ enteritis $(57.6 \%$ vs. $21.1 \%, P=0.005)$ and grade $3-4$ enteritis $(21.2 \%$ vs. $3.8 \%, P=$ 0.033 ) between the two groups. Other acute toxicity reactions were not significantly different.

Conclusions: On the basis of clinical findings, we think that mask wearing for cervical cancer patients undergoing daily postoperative radiotherapy seems debatable. More attention should be focused on the impact of mask wearing on acute toxicity.

\section{Introduction}

Globally, cervical cancer was reported to be the most common gynecologic tumor in females between 2010 and 2019 [1]. Because cervical cancer screening was widely applied in Chinese clinical practice, most patients were diagnosed with early-stage cervical cancer. In our institutions, the current standard treatment for patients with early-stage cervical cancer was radical hysterectomy and pelvic lymph node dissection and para-aortic lymph node sampling. Postoperative concurrent chemoradiotherapy (CCRT) has commonly been recommended for patients with high-risk factors including lymph node metastasis, parametrial invasion, and positive resection margins [2,3]. Over the past two years, the coronavirus disease 2019 (COVID-19) has resulted in a major global health threat worldwide. This epidemic has created great challenges and requirements for radiotherapy. To prevent the spread of the virus and reduce its destructiveness, different countries have adopted different epidemic prevention strategies. In our country, in addition to vaccination, the practice of maintaining social distance and wearing masks are effective ways to reduce the disease transmission [4, 5]. However, prior to the COVID-19 pandemic, it was not a standard requirement for cervical cancer patients to wear masks during postoperative radiotherapy. Thus, whether patients wearing masks will have an impact on radiotherapy was unknown. To the best of our knowledge, no previous study has evaluated the impact of mask wearing on postoperative radiotherapy in patients with early-stage cervical cancer.

The purpose of this study was to retrospectively evaluate the effect of mask wearing on the toxicity of postoperative radiotherapy in patients with cervical cancer during the COVID-19 pandemic. 


\section{Materials And Methods}

\section{Patients}

Between December 2018 and June 2021, the medical data of patients in our institutions who were classified as FIGO stage IB-IIA disease were retrospectively reviewed. The inclusion criteria were as follows: negative nucleic acid detection of COVID-19; FIGO stage IB-IIA cervical cancer; received radical surgery; pathologically confirmed any of the following high-risk factors: lymph node metastasis, parametrial invasion, or positive resection margin; and treated with postoperative CCRT. The policy of wearing a mask was required for every person entering hospitals since the beginning of the COVID-19 pandemic after December 2019. The patients were required to wear a mask, which included surgical masks or $\mathrm{N} 95$ respirators, during the entire radiotherapy procedure and at every visit. According to the Diagnosis and Treatment Protocol for Novel Coronavirus Pneumonia (Trial Version Seven), radiotherapy was suspended or postponed for patients with fever. The patients were required to undergo nucleic acid tests once a week. When the result of nucleic acid detection was negative, patients could continue radiotherapy. The study was approved by the Institution Ethics Committee. All medical information was anonymous, and informed consent was waived due to the retrospective nature of this study.

\section{Radiotherapy and chemotherapy}

Patients (mask wearing, freely breathing) underwent computer tomography (CT) simulation in the supine position using intravenous and oral contrast agents. A cylindrical radio-opaque virginal marker was used before CT simulation. The rectum of patients remained empty during CT simulation and daily treatment. In an attempt to achieve day-to-day reproducibility in bladder filling, the patients were asked to empty the bladder and then asked to drink $500 \mathrm{ml}$ of water 1 hour prior to the planning CT scan and each external beam radiotherapy treatment fraction. External beam radiation therapy was applied using an intensitymodulated radiotherapy (IMRT) technique, including conventional fixed-field IMRT (FF-IMRT) or helical tomotherapy (HT). The clinical target volume (CTV) covered regional lymph node regions (obturator, common iliac, internal iliac, external iliac, and presacral regions) and the upper $2.0 \mathrm{~cm}$ of the vagina and paravaginal soft tissue lateral to the vagina. The external, internal, and common iliac nodal volumes were based on the contrast-enhanced vessels with a $0.7-$ to $1-\mathrm{cm}$ circumferential margin. The planning target volume (PTV) was defined as the CTV expanded to a 0.8- to 1-cm margin with FF-IMRT and a 0.6- to 0.8$\mathrm{cm}$ margin with HT. The pelvic field was prescribed $50.4 \mathrm{~Gy}$ in 28 fractions. Weekly cone-beam CT procedures were conducted for patients with FF-IMRT, and daily on-board megavoltage CT procedures were conducted for patients who were received $\mathrm{HT}$ before treatment. High-dose rate (HDR) intracavitary brachytherapy was administered to patients with a positive resection margin or if the tumor was adjacent $(<5 \mathrm{~mm})$ to the surgical margin of the vagina. The prescription dose was $20 \mathrm{~Gy} / 4$ fractions and was delivered to a depth of $5 \mathrm{~mm}$ below the vaginal mucosa.

The common concurrent chemotherapy regimen was cisplatin $40 \mathrm{mg} / \mathrm{m}^{2}$ weekly for 4-6 cycles. Some patients with renal comorbidities received paclitaxel $60 \mathrm{mg} / \mathrm{m}^{2} /$ week. 


\section{Follow-up and statistical analysis}

After treatment completion, all patients were evaluated after 1 month, followed by evaluations at 3-month intervals for 2 years. The routine follow-up evaluation included gynecological examination, laboratory testing, and abdomen and pelvic MRI/CT. Acute toxicities, measured from the initiation of treatment to 90 days after completion, were graded according to the National Cancer Institute's Common Terminology Criteria for Adverse Events, version 3.0. Statistical analysis was performed using Statistical Package for Social Sciences, version 26.0 (SPSS, Chicago, IL). The chi-square test and the Fisher's exact test were used to compare the toxicity of different groups. Continuous variables were analyzed using the Student's $t$-test or the Mann-Whitney $U$ test. A level of $P<0.05$ was considered to be significant.

\section{Results}

The clinical and pathological characteristics of the patients were shown in Table I. The characteristics were not significantly different between the two groups. Among the 85 patients eligible for the study, 33 patients (38.9\%) wore masks, whereas the other 52 patients $(61.1 \%)$ did not wear masks. The majority of patients (30 patients, $90.9 \%$ ) wore surgical masks, and 3 patients $(9.1 \%)$ wore N95 respirators.

The details of the acute toxicities were shown in Table 2. Anemia was the most common hematologic toxicity. The incidence of grade 3-4 anemia in patients with and without masks was $12.1 \%$ and $11.5 \%$, respectively. In addition, the patients with masks had a lower incidence of Grade 1-2 (27.3\% vs. 34.6\%) leukopenia and Grade $1-2$ (21.2\% vs. $26.9 \%$ ) neutropenia, although these differences were not statistically significant. Cystitis was the most common genitourinary (GU) toxicity, with no patients reporting Grade 34 toxicities. The incidences of acute GU adverse events, hematologic toxicity, dermatitis and postsurgical edema did not differ significantly between the two groups. Proctitis was the most common gastrointestinal (GI) toxicity, with most patients reporting Grade 1-2 toxicities. However, there was a statistically significant difference in the incidence of Grade $1-2$ enteritis $(57.6 \%$ vs. $21.1 \%, P=0.005)$ and Grade $3-4$ enteritis $(21.2 \%$ vs. $3.8 \%, P=0.033)$ between the two groups, respectively.

\section{Discussion}

Early-stage cervical cancer can be cured in approximately $80 \%$ of cases with either radical surgery or radiotherapy [6]. Studies had reported that postoperative CCRT reduced the risk of locoregional recurrence and improved the overall survival rate in patients with high-risk factors after radical surgery $[2$, 7]. However, the combination of surgery and postoperative pelvic radiotherapy had led to observable acute and chronic toxicities, which had aroused the concern and anxieties of physicians. In practice, GI complications had been described as a major toxic consequence of surgery combined with adjuvant radiotherapy for gynecologic malignancy $[8,9]$. IMRT delivered a highly conformal dose to the target volume, with relative sparing of organs at risk. Previous studies had shown that IMRT dramatically reduced the incidence of $\mathrm{GI}$ toxicity in postoperative uterine cancer patients $[10,11]$. Reductions in the volumes of small bowel irradiation might reduce the incidences of acute and late $\mathrm{Gl}$ toxicity [12]. Roeske 
et al.[13] observed that the incidence of acute GI toxicity was significantly correlated with the volume of the small intestine receiving 33.75 Gy or more. After hysterectomy, the pelvis usually contains a large amount of the small intestine, and the positions of the small intestine during treatment might differ from their positions at the time of simulation and planning. In the past two special years, it has become normal for patients to wear masks during radiotherapy due to the COVID-19 pandemic. Although alveolar ventilation/gas exchange was not significantly impacted by face masks, females have smaller lungs and rib cages and disproportionally smaller large conducting airways, which increased their effort in breathing when wearing masks $[14,15]$. Because of the increase of in respiratory resistance, the abdominal breathing movements of patients were obvious during supine radiotherapy, resulting in an increase in small intestine activity, and more of the small intestine received more radiation doses than expected. In our study, the patients who wore masks had a higher incidence of Grade $1-2$ enteritis (57.6\% vs. $21.1 \%, P$ $=0.005)$ and Grade $3-4$ enteritis $(21.2 \%$ vs. $3.8 \%, P=0.033)$ than the patients who did not wear masks. The results supported our hypothesis that wearing masks had a significant effect on small intestinal toxicity.

The position and volume of the bladder and rectum were mainly affected by their filling [16]. Our study showed that the incidences of acute GU toxicity, acute hematological toxicity, edema and dermatitis did not differ significantly between the two groups. In addition, the patients wearing masks had a lower incidence of Grade 1-2 (27.3\% vs. $34.6 \%$ ) leukopenia and Grade 1-2 (21.2\% vs. $26.9 \%)$ neutropenia, although this difference was not statistically significant. Wearing masks not only reduced the spread and infection of COVID-19 but also avoided the infection of influenza-like illness and laboratory-confirmed influenza [17], which might result in slight improvement in the health of patients and partly explain the reduced the incidence of agranulocytosis.

Our study had a few limitations. This was a retrospective single institute study, and it was not feasible to completely exclude the potential for selection bias from this study. Second, the sample sizes were small.

Because the follow-up time was short, we only analyzed treatment-related acute toxicity and not analyzed chronic toxicity. Third, we did not consider the impact of vaccination, which potentially influenced the conclusions of our study. Fourth, a previous study showed that N95 respirators provide the greatest amount of protection but also have greater resistance to breathing than surgical masks [18]. Because only 3 patients wore N95 respirators, we did not analyze the different effects of surgical masks and N95 respirators on acute toxicity. Fifth, the physicians did not reach a consensus on the definition and outline of the small intestinal structure in our institutions, and we did not make a comparative analysis of dosimetry. Last, some patients received treatment in the outpatient department, and doctors did not record the patient's relevant medication history and mild comorbidities in time. We hope to have more multicenter prospective randomized controlled trials to verify our findings.

\section{Conclusion}

Based on our study, mask wearing in patients undergoing daily postoperative radiotherapy had a significant effect on small intestinal acute toxicity. After the COVID-19 pandemic, wearing masks should 
not be part of routine radiotherapy.

\section{Abbreviations}

CCRT: concurrent chemoradiotherapy; COVID-19: coronavirus disease 2019; FIGO: Federation of gynecology and obstetrics; CT: computer tomography; IMRT: intensity-modulated radiotherapy; FF-IMRT: fixed-field intensity-modulated radiotherapy; HT: helical tomotherapy; CTV: clinical target volume; PTV: planning target volume; HDR: High-dose rate; GU: genitourinary; GI gastrointestinal.

\section{Declarations}

\section{Acknowledgements}

Not applicable

\section{Authors' contributions}

GZ collected and organized the data, wrote the manuscript. CW, CM, LM, YZ collected and organized the data. CF designed the study and reviewed the manuscript. All authors read and approved the final manuscript.

\section{Funding}

Not applicable

\section{Availability of data and materials}

The datasets used and/or analyzed for the present study are available from the corresponding author on reasonable request.

\section{Ethics approval and consent to participate}

Study protocol was approved be the Institution Ethics Committee

\section{Consent for publication}

Not applicable.

\section{Competing interests}

The authors declare that they have no competing interests.

\section{References}


1. Kocarnik JM, Compton K, Dean FE, Fu W, Gaw BL, Harvey JD, et al. Cancer Incidence, Mortality, Years of Life Lost, Years Lived With Disability, and Disability-Adjusted Life Years for 29 Cancer Groups From 2010 to 2019: A Systematic Analysis for the Global Burden of Disease Study 2019. JAMA Oncol. 2021.

2. Peters WA 3rd, Liu PY, Barrett RJ 2nd, Stock RJ, Monk BJ, Berek JS, et al. Concurrent chemotherapy and pelvic radiation therapy compared with pelvic radiation therapy alone as adjuvant therapy after radical surgery in high-risk early-stage cancer of the cervix. J Clin Oncol. 2000;18:1606-13.

3. Kim D, Ki Y, Kim W, Park D, Lee J, Lee J, et al. Adjuvant external beam radiation and brachytherapy for vaginal resection margin positive cervical cancer. Radiat Oncol J. 2018;36:147-52.

4. Jiang T, Wang H, Gao GF, Jiang X. Wearing Face Masks - the Simple and Effective Way to Block the Infection Source of COVID-19. China CDC Wkly. 2020;2:268-9.

5. Wang J, Pan L, Tang S, Ji JS, Shi X. Mask use during COVID-19: A risk adjusted strategy. Environ Pollut. 2020;266:115099.

6. Landoni F, Maneo A, Colombo A, Placa F, Milani R, Perego P, et al. Randomised study of radical surgery versus radiotherapy for stage Ib-lla cervical cancer. Lancet. 1997;350:535-40.

7. Monk BJ, Wang J, Im S, Stock RJ, Peters WA 3rd, Liu PY, et al. Rethinking the use of radiation and chemotherapy after radical hysterectomy: a clinical-pathologic analysis of a Gynecologic Oncology Group/Southwest Oncology Group/Radiation Therapy Oncology Group trial. Gynecol Oncol. 2005;96:721-8.

8. Barter JF, Soong SJ, Shingleton HM, Hatch KD, Orr JW Jr. Complications of combined radical hysterectomy-postoperative radiation therapy in women with early stage cervical cancer. Gynecol Oncol. 1989;32:292-6.

9. Montz FJ, Holschneider CH, Solh S, Schuricht LC, Monk BJ. Small bowel obstruction following radical hysterectomy: risk factors, incidence, and operative findings. Gynecol Oncol. 1994;53:114-20.

10. Chen MF, Tseng CJ, Tseng CC, Kuo YC, Yu CY, Chen WC. Clinical outcome in posthysterectomy cervical cancer patients treated with concurrent Cisplatin and intensity-modulated pelvic radiotherapy: comparison with conventional radiotherapy. Int J Radiat Oncol Biol Phys. 2007;67:1438-44.

11. Isohashi F, Mabuchi S, Yoshioka Y, Seo Y, Suzuki O, Tamari K, et al. Intensity-modulated radiation therapy versus three-dimensional conformal radiation therapy with concurrent nedaplatin-based chemotherapy after radical hysterectomy for uterine cervical cancer: comparison of outcomes, complications, and dose-volume histogram parameters. Radiat Oncol. 2015;10:180.

12. Beriwal S, Gan GN, Heron DE, Selvaraj RN, Kim H, Lalonde R, et al. Early clinical outcome with concurrent chemotherapy and extended-field, intensity-modulated radiotherapy for cervical cancer. Int J Radiat Oncol Biol Phys. 2007;68:166-71.

13. Roeske JC, Bonta D, Mell LK, Lujan AE, Mundt AJ. A dosimetric analysis of acute gastrointestinal toxicity in women receiving intensity-modulated whole-pelvic radiation therapy. Radiother Oncol. 2003;69:201-7. 
14. Fikenzer S, Uhe T, Lavall D, Rudolph U, Falz R, Busse M, et al. Effects of surgical and FFP2/N95 face masks on cardiopulmonary exercise capacity. Clin Res Cardiol. 2020;109:1522-30.

15. Dominelli PB, Molgat-Seon Y, Sheel AW. Sex Differences in the Pulmonary System Influence the Integrative Response to Exercise. Exerc Sport Sci Rev. 2019;47:142-50.

16. Jadon R, Pembroke CA, Hanna CL, Palaniappan N, Evans M, Cleves AE, et al. A systematic review of organ motion and image-guided strategies in external beam radiotherapy for cervical cancer. Clin Oncol (R Coll Radiol). 2014;26:185-96.

17. Loeb M, Dafoe N, Mahony J, John M, Sarabia A, Glavin V, et al. Surgical mask vs N95 respirator for preventing influenza among health care workers: a randomized trial. JAMA. 2009;302:1865-71.

18. Hopkins SR, Dominelli PB, Davis CK, Guenette JA, Luks AM, Molgat-Seon Y, et al. Face Masks and the Cardiorespiratory Response to Physical Activity in Health and Disease. Ann Am Thorac Soc. 2021;18:399-407.

\section{Tables}

Table 1. The patients and clinical characteristics classified by mask-wearing 


\begin{tabular}{|c|c|c|c|c|}
\hline Factors & $\begin{array}{l}\text { Cases } \\
(\%)\end{array}$ & $\begin{array}{l}\text { CCRT with mask wearing }(n= \\
\text { 33) }\end{array}$ & $\begin{array}{l}\text { CCRT } \\
(n=52 \\
(n=52\end{array}$ & $\begin{array}{l}\text { P value } \\
(\mathrm{p}[0.05)\end{array}$ \\
\hline \multicolumn{5}{|l|}{ Age (year) } \\
\hline Median & 50 & 49 & 54 & 0.234 \\
\hline \multicolumn{5}{|l|}{ FIGO stage } \\
\hline IB & $60(70.6)$ & 17 & 43 & 0.481 \\
\hline IIA & $25(29.4)$ & 16 & 9 & \\
\hline \multicolumn{5}{|l|}{ Histological type } \\
\hline squamous cell carcinoma & $60(71)$ & 26 & 34 & 0.102 \\
\hline adenocarcinoma & $17(20)$ & 5 & 12 & \\
\hline $\begin{array}{l}\text { adenosquamous } \\
\text { carcinoma }\end{array}$ & $8(9)$ & 2 & 6 & \\
\hline \multicolumn{5}{|l|}{ DSI } \\
\hline$\geq 50 \%$ & $67(78.8)$ & 23 & 44 & 0.236 \\
\hline$\bigotimes 50 \%$ & $18(21.2)$ & 10 & 8 & \\
\hline \multicolumn{5}{|l|}{ LVSI } \\
\hline$(+)$ & $46(54.1)$ & 10 & 36 & 0.805 \\
\hline$(-)$ & $39(45.9)$ & 23 & 16 & \\
\hline \multicolumn{5}{|l|}{ Primary tumor size } \\
\hline$\geq 4 \mathrm{~cm}$ & $35(41.2)$ & 14 & 21 & 0.422 \\
\hline$\nabla 4 \mathrm{~cm}$ & $50(58.8)$ & 19 & 31 & \\
\hline \multicolumn{5}{|l|}{ Lymph nodes metastasis } \\
\hline$(+)$ & $64(75.3)$ & 21 & 43 & 0.174 \\
\hline$(-)$ & $21(24.7)$ & 12 & 9 & \\
\hline \multicolumn{5}{|l|}{ Parametrial invasion } \\
\hline$(+)$ & $48(56.4)$ & 15 & 33 & 0.081 \\
\hline$(-)$ & $37(43.5)$ & 17 & 20 & \\
\hline \multicolumn{5}{|l|}{ Positive resection margin } \\
\hline$(+)$ & $2(2.3)$ & 0 & 2 & 1 \\
\hline$(-)$ & $83(97.7)$ & 33 & 50 & \\
\hline
\end{tabular}




\begin{tabular}{|c|c|c|c|c|}
\hline \multicolumn{5}{|c|}{ HDR brachytherapy } \\
\hline Yes & $3(3.5)$ & 1 & 2 & 0.976 \\
\hline No & $82(96.5)$ & 32 & 50 & \\
\hline \multicolumn{5}{|c|}{ Hemoglobin } \\
\hline$\geq 110 \mathrm{~g} / \mathrm{L}$ & $78(91.8)$ & 30 & 48 & 0.391 \\
\hline$\otimes 110 \mathrm{~g} / \mathrm{L}$ & $7(8.2)$ & 3 & 4 & \\
\hline \multicolumn{5}{|c|}{ Concurrent Chemotherapy } \\
\hline Cisplatin & $81(95.3)$ & 31 & 50 & 0.308 \\
\hline Paclitaxel & $4(4.7)$ & 2 & 2 & \\
\hline
\end{tabular}

CCRT, concurrent chemoradiotherapy; FIGO, International Federation of Gynecology and Obstetrics; DSI, deep stromal invasion; LVSI, lymphovascular space invasion ;SCC-Ag, squamous cell carcinoma antigen; HDR, high-dose-rate

Table 2. Acute toxicity of patients treated by CCRT with mask-wearing and CCRT 


\begin{tabular}{|c|c|c|c|c|c|}
\hline \multirow[t]{2}{*}{ Toxicity } & \multicolumn{2}{|c|}{ CCRT with mask wearing } & \multicolumn{2}{|c|}{ CCRT } & \multirow{2}{*}{$\begin{array}{l}\text { P value } \\
\text { (p[0.05) }\end{array}$} \\
\hline & \multicolumn{2}{|c|}{ Number Percentage } & \multicolumn{2}{|c|}{ Number Percentage } & \\
\hline \multicolumn{6}{|c|}{ Hematological toxicity } \\
\hline \multicolumn{6}{|c|}{ Leukopenia } \\
\hline Grade 1-2 & 9 & $27.3 \%$ & 18 & $34.6 \%$ & NS \\
\hline Grade 3-4 & 6 & $18.2 \%$ & 9 & $17.3 \%$ & NS \\
\hline \multicolumn{6}{|c|}{ Neutropenia } \\
\hline Grade 1-2 & 7 & $21.2 \%$ & 14 & $26.9 \%$ & NS \\
\hline Grade 3-4 & 2 & $6.1 \%$ & 5 & $9.6 \%$ & NS \\
\hline \multicolumn{6}{|c|}{ Thrombocytopenia } \\
\hline Grade 1-2 & 18 & $54.5 \%$ & 27 & $51.9 \%$ & NS \\
\hline Grade 3-4 & 0 & 0 & 0 & 0 & NA \\
\hline \multicolumn{6}{|l|}{ Anemia } \\
\hline Grade 1-2 & 23 & $69.7 \%$ & 37 & $71.2 \%$ & NS \\
\hline Grade 3-4 & 4 & $12.1 \%$ & 6 & $11.5 \%$ & NS \\
\hline \multicolumn{6}{|c|}{ Gastrointestinal toxicity } \\
\hline \multicolumn{6}{|l|}{ Proctitis } \\
\hline Grade 1-2 & 26 & $78.7 \%$ & 39 & $75 \%$ & NS \\
\hline Grade 3-4 & 2 & $6.1 \%$ & 2 & $3.8 \%$ & NS \\
\hline \multicolumn{6}{|l|}{ Diarrhea } \\
\hline Grade 1-2 & 14 & $42.4 \%$ & 23 & $44.2 \%$ & NS \\
\hline Grade 3-4 & 1 & $3 \%$ & 0 & 0 & NS \\
\hline \multicolumn{6}{|l|}{ Enteritis } \\
\hline Grade 1-2 & 19 & $57.6 \%$ & 11 & $21.1 \%$ & 0.005 \\
\hline Grade 3-4 & 7 & $21.2 \%$ & 2 & $3.8 \%$ & 0.033 \\
\hline \multicolumn{6}{|c|}{ Genitourinary toxicity } \\
\hline \multicolumn{6}{|l|}{ Cystitis } \\
\hline Grade 1-2 & 11 & $33.3 \%$ & 16 & $30.8 \%$ & NS \\
\hline Grade 3-4 & 0 & 0 & 0 & 0 & NA \\
\hline
\end{tabular}




\begin{tabular}{|c|c|c|c|c|c|}
\hline \multicolumn{6}{|l|}{ Frequency } \\
\hline Grade 1-2 & 3 & $9.1 \%$ & 5 & $9.6 \%$ & NS \\
\hline Grade 3-4 & 1 & $3 \%$ & 2 & $3.8 \%$ & NS \\
\hline \multicolumn{6}{|c|}{ Postsurgical edema } \\
\hline Grade 1-2 & 4 & $12.1 \%$ & 9 & $17.3 \%$ & NS \\
\hline Grade 3-4 & 0 & 0 & 0 & 0 & NA \\
\hline \multicolumn{6}{|l|}{ Dermatitis } \\
\hline Grade 1-2 & 13 & $39.3 \%$ & 18 & $34.6 \%$ & NS \\
\hline Grade 3-4 & 0 & 0 & 0 & 0 & NA \\
\hline
\end{tabular}

CCRT, concurrent chemoradiotherapy; NS, not significant.; NA, not applicable. 